\title{
Review
}

\section{Physiology of Starvation and Refeeding Syndrome}

\author{
Raghuveer Choudhary ${ }^{1}$ Vinode Kumar Chawala², Jayant Kumar ${ }^{3}$, Raj Kumar Rathore ${ }^{4}$
}

\begin{abstract}
Refeeding syndrome represents a series of metabolic complications that sometimes results by provision of nutrients, primarily carbohydrate, to a patient in a nutritionally compromised state. Refeeding syndrome is associated with hypophosphatemia, hypokalemia and hypomagnesaemia, fluid retention and vitamin deficiencies including thiamin are also of concern. If severe, refeeding syndrome may result in respiratory, cardiac, and neuromuscular dysfunction, especially in the stressed, elderly, or severely malnourished patient. This article discuss the physiology of starvation and the pathophysiology behind refeeding syndrome, identify the patients at greatest risk, and provide practical tips for prevention. As increased awareness and understanding of refeeding syndrome may help, prevent further such incidents and clinicians learn to recognize patients at risk and avoid overaggressive nutrition support regimes.
\end{abstract}

Key words: Starvation, Refeeding Syndrome

J Bangladesh Soc Physiol. 2010 December; 5(2): 101-110 For author affiliations, see end of text.

http://www.banglajol.info/index.php/JBSP

\section{Introduction}

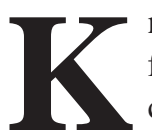

nowledge of the dangers of refeeding famine victims dates back many centuries. Historically, problems such

as cardiac dysfunction, edema and neurological changes were observed when malnourished refugees were suddenly provided with adequate food. Such problems have also been observed when relief efforts provide food to victims of famine. After world war-II a number of investigators reported the unexpected death of starved prisoners of war (POW) shortly after reintroduction of high-caloric, high-carbohydrate food. Autopsies on those individuals did not reveal any obvious cause. ${ }^{1-3}$

These potentially life threatening refeeding complications became apparent once again in the 1970's and 1980's with the widespread use of total parenteral nutrition (TPN) in hospitalized patients who were often malnourished ${ }^{4}$.

An increased awareness and understanding of Refeeding Syndrome may help to prevent further such incidents as clinicians learn to recognize patients at risk and avoid over aggressive nutrition support regimes.

\section{Incidence of refeeding syndrome}

The incidence of Refeeding Syndrome is unknown. In studies of patients on total parenteral nutrition (TPN), the incidence of hypophosphatemia ranged from 30\%-38\% when phosphorous was provided in the solution, to 100\% when TPN without phosphorous was administered $^{5}$. In cancer patients, it has been reported as high as $25 \%{ }^{6}$. It is more common in the elderly, although it may be masked by comorbid conditions ${ }^{7}$.

There are no reports of randomized clinical trials, controlled trials looking at the incidence of Re feeding Syndrome itself. Mild symptoms often go unnoticed or may be attributed to other cause. Conversely, low serum levels of phosphorus, potassium and magnesium thought to be due to Refeeding Syndrome may be due to other factors including decreased dietary intake, insufficient gastrointestinal absorption, increased renal or nonrenal losses and transcellular shifts. (Table-I). 
Starvation and Refeeding

Review

Table I: Potential Causes of Hypokalemia, Hypophosphatemia, and Hypomagnesemia ${ }^{22}$

(A) Hypophosphatemia

\begin{tabular}{lll}
\hline General & Metabolic & Medications \\
Malabsorption & Hypomagnesemia & Diuretics \\
Glucose administration & Hypokalemia & Beta-agonists \\
vitamin D deficiency & Hypocalcemia & Epinephrine \\
Volume repletion & systemic alkalosis & Insulin \\
Rhabdomyolysis & Hyperparathyroidism & Antacids \\
Hemodialysis & Hyperaldosteronism & Carafate \\
Initiation of CVVHD & & Glucagon \\
Diabetic ketoacidosis & & Bicarbonate \\
Alcoholism & & Corticosteroids \\
Sepsis & & Cisplatin \\
Gram-negative bacteremia & & Theophylline \\
Refeeding Syndrome & \\
\hline
\end{tabular}

(B) Hypokalemia

\begin{tabular}{lll}
\hline General & Metabolic & Medications \\
Vomiting / Diarrhea & Hypomagnesemia & AlbuterolDiuretics \\
Insulin delivery & Metabolic Alkalosis & Insulin \\
Refeeding syndrome & & Carbenicillin \\
& & Terbutaline \\
& & Ticarcillin \\
\hline
\end{tabular}

\section{(C) Hypomagnesemia}

\begin{tabular}{lll}
\hline General & Metabolic & Medications \\
Diarrhea & Diabetes Mellitus & Amphotericin B \\
Malabsorption & & Cisplatin \\
Alcoholism & Cyclosporin \\
Refeeding Syndrome & Ticarcillin \\
& Carbenicillin \\
& Digoxin \\
& Alcohol \\
& Gentamycin \\
& Insulin \\
\hline
\end{tabular}

What is refeeding syndrome?

Re feeding Syndrome represents a host of metabolic complications that sometimes results when 
feeding a malnourished patient. By definitions, Re feeding Syndrome describes a potentially fatal medical condition that may affect malnourished/ ill patients in response to an inappropriately high protein-caloric intake. This commonly occurs following the institution of nutritional support, either parenteral or enteral or unrestricted oral intake.

Numerous Physiological changes occur during refeeding that may result in increased patient morbidity and mortality. Intracellular mineral depletion (eg. hypophosphatemia, hypomagnesemia, hypokalemia), body fluid disturbances (refeeding edema),vitamin deficiencies (eg. thiamine), life threatening cardiac arrhythmias, respiratory arrest and congestive heart failure are among the most often reported events associated with refeeding. ${ }^{4,8}$ Death may have been avoided by recognition of the patients risk for Refeeding Syndrome, appropriate nutritional support management, effective monitoring, and therapy for complications.

\section{Physiology of starvation}

Under normal conditions, glucose is the body's preferred fuel. Glucose is primarily derived from carbohydrates in the diet. The body depends on a consistent intake of carbohydrate for continued glucose supply. Glucose is available in the blood stream for fuel for 2-3 hours after ingestion and can be stored as glycogen, \& 300gms of glucose are stored as glycogen in the liver and muscles. In absence of oral intake, glycogen can provide energy up to 24 hours. A minimum of 100-150 gm of glucose per day is needed to provide adequate glucose to the brain and to prevent protein breakdown for glucose production. ${ }^{10}$

When adequate glucose is available, protein is used for non-fuel functions, such as building muscle and the synthesis of enzymes, hormones, anti-bodies and transport proteins. Carbohydrate, protein and fat intake in excess of needs is stored as fat. Fat is the primary storage fuel in the human body.
During the short period of fasting (upto 24 hours.) obligatory glucose is obtained by glycogenolysis. After depletion of glycogen stores, amino-acids are mobilized from muscle and fatty acids are mobilized from adipose tissues. Both are degraded further to produce glucose and glycerol respectively from gluconeogenesis. Glucose is also produced via gluconeogenesis from lactate and pyruvate. The initial period of starvation is marked by increased protein catabolism. As the nutrient deprivation is prolonged, the rate of proteolysis decreases as the body further adapts to the decreased nutrient intake, basal metabolic rate slows by as much as $20 \%-25 \%{ }^{11}$. Peripheral tissues and most organs can adapt to using fatty acids as a fuel source. The brain, as a primary end user of glucose, switches over to Ketones as a partial fuel source and decreases it's reliance on glucose as a substrate. These adaptive mechanisms, in the non-stressed state, decrease the need for glucose, thereby sparing muscle mass.

Prolonged starvation leads to loss of lean body mass, adipose tissue and fluid. Eventually, starvation also affects the visceral protein mass and the function of vital organs. Respiratory function may decline due to respiratory muscle wasting which may limit $\mathrm{Co}_{2}$ excretion ${ }^{12}$. Cardiac mass and output are also decreased as well. In the absence of additional stressors, such as illness or injury, serum protein levels are maintained at relatively normal levels during pure protein, calorie malnutrition. A severely malnourished patient may have a normal albumin level despite prolonged starvation. This is due in part to decreased break down of serum proteins and to extravasations of fluid into the interstitial space that may make serum levels appear falsely elevated. In fact, it is not uncommon for a severely malnourished patient to initially loose weight after nutrition support is started due to diuresis of this additional extracellular fluid. (Table II) 
Table II: Pathophysiology of Starvation

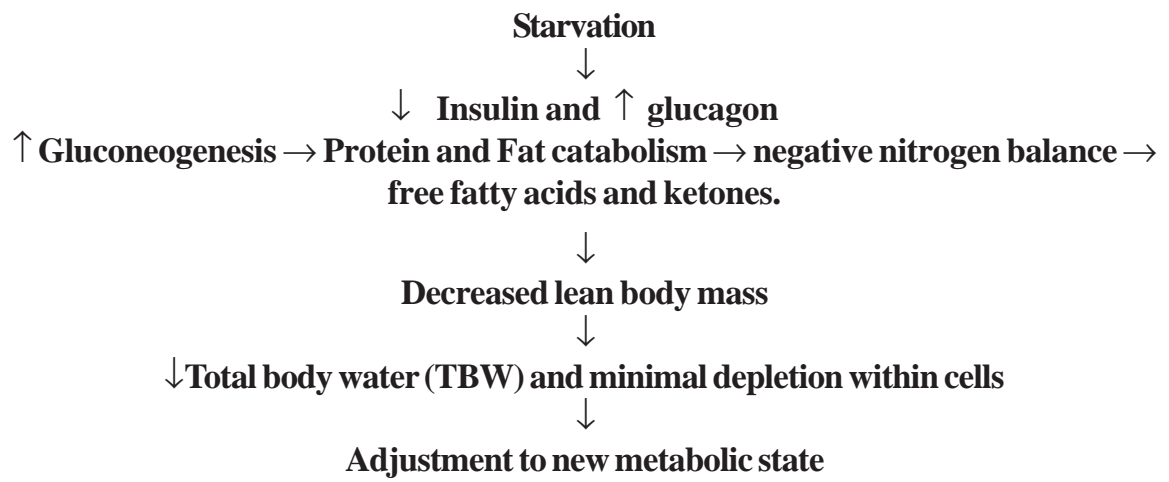

During starvation there is a change in the hormonal milieu in addition to a reduction in the basal metabolic rate, conservation of proteins, prolongation of organ function, preferential catabolism of skeletal muscles and loss of visceral cell mass ${ }^{9}$. The concentration of insulin is deceased whilst glucagon increases resulting in the conversion of glycogen to glucose in addition to gluconeogenesis from lipid and protein stores. Free fatty acids and ketones bodies replace glucose as the primary source of energy ${ }^{8}$

Decreased nutrient intake and poor nutritional status may lead to depleted vitamin and mineral status, including depletion of phosphorus, magnesium and potassium. Unlike glucose metabolism, fatty acid oxidation does not require phosphorous intermediates; hence phosphorus requirements is decreased during starvation.

\section{Metabolic response to refeeding}

There are many consequences that occur when nutrition namely carbohydrate, is provided to a starved patient. (Table III).

Table III: Pathophysiology of Refeeding<smiles></smiles>

Conversion of glucose as major energy source instead of protein and ketones

$\downarrow$

$\uparrow$ requirement for phosphorus for kreb’s cycle

$\downarrow$

$\uparrow$ Insulin release*

$\downarrow$

$\uparrow$ Cellular glucose, calcium, phosphorus, magnesium and total body water uptake.

$\downarrow$

$\uparrow$ Protein synthesis and utilization of thiamine.

*Intracellular shifts of calcium,phosphorus,magnesium and potassium.* Insulin release stimulates the sodium potassium ATPase pump (which requires magnesium as a co-factor). This drives potassium into the cells and moves sodium out. Carbohydrate load and insulin release stimulate phosphate shifts into the cells and phosphate depletion is associated with increased urinary magnesium excretion. This leads to low extracellular phosphate, magnesium and potassium may precipitate the symptoms of Refeeding Syndrome. Thiamine is an essential coenzyme in carbohydrate metabolism. The symptoms of thiamine deficiency e.g. Wernicke's encephalopathy, can be precipitated by feeding with carbohydrate in a vitamin B depleted patient. 
Table IV: Complications associated with refeeding syndrome ${ }^{22}$

\begin{tabular}{lll}
\hline Hypophosphatemia & Hypokalemia & Hypomagnesemia \\
Respiratory & Cardiac Dysfunction & Cardiac Abnormalities \\
- Failure & - Arrhythmia & - Arrhythmias \\
- Ventilator dependency & - Cardiac arrest & - Tachycardia \\
Cardiac & - EKG changes & Neurologic \\
- Arrhythmia & - Digoxin toxicity & - Altered mental status \\
- Congestive heart failure & Neurologic & - Weakness \\
- Cardiomyopathy & - Weakness & - Tetany \\
- Decreased cardiac contractility & - Paralysis & - Paresthesia- Seizures \\
- Hypotension & - Rhabdomyolysis & -Ataxia \\
Skeletal & - Lethargy/confusion & - Vertigo \\
- Rhabdomyolysis & Metabolic & - Tremors \\
- Weakness & - Metabolic alkalosis & Gastrointestinal Problems \\
Neurologic & Gastrointestinal & - Abdominal pain \\
- Altered mental status & - Paralytic ileus & - Diarrhea \\
- Paralysis & - Constipation & - Constipation \\
- Seizures & & - Anorexia \\
Endocrine & & Electrolyte \\
- Insulin resistance & & - Hypokalemia \\
- Osteomalacia & & - Hypocalcemia \\
Hematologic & & Hematologic \\
- Leukocyte dysfunction & & - Anemia \\
- Altered RBC morphology & & \\
- Thrombocytopenia & & \\
- Decreased platelet function & & \\
- Hemolytic anemia & & \\
- Decreased oxygen release from & & \\
oxyhemoglobin & & \\
\hline
\end{tabular}

The driving factor for refeeding complications is insulin secretion. Insulin promotes glucose uptake, along with phosphorus and other electrolytes into the cells. This intracellular shift, along with already depleted electrolyte pool, can lead to dangerously low levels of serum phosphorus, potassium and magnesium (but not necessarily all three). Serious respiratory, cardiac and neurological complications can occur. In most severe cases, they can be fatal (Table IV).

\section{Excess glucose}

Glucose is an important substrate in specialized nutrition support. As previously discussed overfeeding carbohydrates (enteral or parenteral) may result in adverse consequences. Hyperglycemia is a common complication of specialized nutrition support. Critically ill patients are often hypermetabolic and insulin resistant because of the metabolic response to stress and infection. Gluconeogenesis continues in the stressed patient because of hormonal mediators inspite of high circulating levels of insulin. Hyperglycemia develops in non diabetic patients if given large amounts of glucose. This is especially true in the hypercatabolic, stressed state $^{13}$. Uncontrolled hyperglycemia can lead to hyper osmolar non ketotic coma, which has a 
mortality rate as high as $40 \%{ }^{14}$. Hyperglycemia may also place the compromised, critically ill patient at higher risk of the development of sepsis $^{15}$.Serum glucose levels should be kept in the range of $100-200 \mathrm{mg} / \mathrm{dL}^{15}$.

\section{Phosphorus}

Hypophosphatemia is one of the most serious metabolic complications observed in response to specialized nutrition support ${ }^{16,17}$. Plummeting serum phosphorus levels may be seen within 24 to 72 hours after beginning of therapy. However, it is possible to see a delay in the development of hypophosphatemia of 5 to 10 days in patients who have normal body stores of phosphorus. In response to refeeding, there is a movement of inorganic phosphorus from the extracellular to the intracellular space because of utilization of phosphorus for the synthesis of phosphorylated compound. Insulin also promotes the uptake of both phosphorus and glucose in skeletal muscle and liver ${ }^{18}$. Severe hypophosphatemia (below 1.0 $\mathrm{mg} / \mathrm{dL}$ ) is associated with serious life threatening complications involving all major organ systems. ${ }^{1,18}$ It can led to respiratory failure, cardiac abnormalities, central nervous system dysfunction including seizures, red blood cell dysfunction and leukocyte dysfunction ${ }^{4.6 .19}$

One of the potential consequences of hypophospatemia may be decreased oxygen delivery to the cell (ischemia) as hypohosphatemia leads to decreased levels of 2,3 diphosphoglyceride (2, 3 DPG) consequently impairing oxygen release from hemoglobin ${ }^{20,21}$.

\section{Potassium}

Refeeding causes a shift of potassium into cell and may result in hypokalemia if potassium is not adequately supplied in the diet. Potassium moves into cells along with glucose and other energy substrates.

Symptoms of hypokalamia include muscle weakness, paralysis, paralytic ileus, cardiac arrhythmias, cardiac arrest, coronary ischemia, rhabdomyolysis, renal dysfunction, decreased urinary concentrating ability, polyuria, dysuria, constipation, respiratory depression, exacerbation of hepatic encephalopathy. 4,6,19

\section{Magnesium}

Magnesium shifts intracellularly with refeeding and new tissue synthesis. Hypomagnesemia is a frequent complication of both enteral and parenteral nutrition support.

Adequate magnesium is essential for normalizing phosphorus and potassium levels and is also required for over 300 metabolic pathways, including those involving ATP production. Therefore hypophosphatemia and hypokalemia associated with refeeding may be exacerbated by an underlying hypomagenesemia. ${ }^{22}$ Clinical deficiency symptoms are associated with serum level below 1.0mEq/L. Hypomagnesemia may lead to arrhythmias, tachycardia, respiratory depression, abdominal pain, diarrhea, ataxia, confusion, muscle tremors, weakness and tetany. 4,6,19

\section{Thiamine}

While not a hall mark of Re feeding Syndrome, thiamin deficiency can still be of significant importance to the malnourished patient at risk for refeed. Thiamine $\left(B_{1}\right)$ is a water soluble vitamin with a biological half life of $91 / 2$ to $181 / 2$ days $^{23}$. In patients with suboptimal intake, it estimated that deficiency can occur in less than 28 days. ${ }^{12}$

Thiamin is the precursor for the active metabolite thiamin pyrophosphate (TPP), essential for optimal glucose utilization and metabolism. TPP is a co-factor for three critical enzymes, in particular, pyruvate dehydrogenase. In thiamin deficiency, the conversion of pyruvate to acetyl coenzyme A (CoA) is blocked and the accumulating pyruvate is subsequently converted to lactate. This results in an overproduction of lactate and lactic acidosis follows. Inadequate levels of thiamin can also precipitate Wernicke's Encephalopathy (WE) in malnourished patients. Glucose load, whether delivered enterally or parenterally, will increase the demand for thiamin. Thiamine depletion may be exacerbated by dialysis, diuretics, diarrhea, alcohol, folate deficiency or malabsorption. 24-,26

The 2004 guidelines of the British Association of Pharmacology recommend a minimum of 500mg. IV TID for a minimum of two days, followed by 
Review

500mg IV daily for five days for patients with suspected or diagnosed WE. ${ }^{27}$

\section{Fluid retention}

Insulin secretion associated with sudden carbohydrate infusion also lead to sodium fluid retention. This is thought to be due to insulin's effect on renal tubules leading to anti natriuresis. This antidiuretic effect causes an increase in extracelluler fluid volume. Therefore, excessive sodium containing fluid infusion can lead to edema or, in severe cases, the combination of increased extracellular fluid volume and the cardiomyopathy often present in severely malnourished individuals \& can lead to pulmonary edema, respiratory compromise and cardiac failure.

\section{Prevention of the refeeding syndrome}

The key to prevent the Refeeding Syndrome is to be aware of it and it's consequences. Prevention begins with recognition of those patients at greatest risk of the development of potentially lethal complications from rapid overzealous feeding.

\section{Initial assessment of refeeding syndrome:}

Your patient is at risk of refeeding syndrome if there is: History of chronic malnutrition; acute weight loss of greater than $10 \%$ of pre-morbid body weight; little or no nutritional intake of any form for greater than 7-10 days.

\section{The risks of refeeding syndrome are higher in patients with}

Significant co-morbidity, e.g. infection, surgery, pressure sores, cancer; evidence of physiological stress, e.g. postoperatively or patients who require critical care input; prolonged fasting; anorexia nervosa; chronic alcoholism; electrolyte abnormalities (Potassium/Magnesium/Phosphate); the elderly (due to the increased risk of malnutrition in this patient group).

\section{History and clinical examination}

To record weight, rate of weight loss, dietary intake, reasons for malnutrition - physiological
Starvation and Refeeding

impediment to oral or parenteral intake, swallowing difficulties, impaired intestinal function, heart rate, pulse rate, blood pressure,

respiratory rate and level of consciousness- 6 hourly for first 72 hours, daily temperature, ECG if patient has:

Irregular pulse, abnormal heart rate, serum potassium or phosphate level below normal range)

If evidence of cardiac abnormalities is present on assessment or during re feeding, patient will require cardiac monitoring. If necessary, transfer to ICU

\section{Initial management of refeeding syndrome}

Fluid resuscitation and monitoring fluid balance

Assess and carefully restore circulatory volume, monitor pulse rate, fluid intake and output. Malnourished patients have a reduced tolerance of intravenous fluid in moderate to high intake (i.e. more than 2 liters per $24 \mathrm{hrs}$.) can lead to heart failure.

Administration of intravenous fluids may be necessary in the initial 72 hours until sufficient oral intake is achieved.

If evidence of dehydration, for careful rehydration i.e. 1-2 liters in the first 24 hrs depending on response. Greater volumes only if severely dehydrated.

Total fluid intake (including i.v., enteral and oral) should aim for a maximum of $30 \mathrm{ml} / \mathrm{kg}$ per day (i.e. commonly 1.5 liter or less).

At least 6 hourly monitoring of blood pressure, pulse and respiratory rate is necessary to detect evidence of heart failure or inadequate intra vascular volume.

\section{Correction of electrolyte abnormalities}

Ensure recent (last 48 hours) electrolyte levels are available. These should include: urea and electrolytes $\left(\mathrm{Na}^{+}, \mathrm{K}^{+}\right)$, Phosphate, Magnesium, Calcium (add to standard blood profile) liver function tests, full blood counts. If electrolytes are deranged consider to treat possible cause. 
Starvation and Refeeding

Review

Suggested guidelines for phosphorus replacement in adult I.V. ${ }^{28,29,30}$

\begin{tabular}{|c|c|c|}
\hline Serum $\mathrm{Po}_{4}$ Level (mg/dL) & $\begin{array}{l}\text { Recent Uncomplicated } \\
\mathrm{mmol} / \mathrm{kg} \text {. of } \mathrm{Po}_{4}\end{array}$ & $\begin{array}{l}\text { Prolonged or Complicated } \\
\mathrm{mmol} / \mathrm{kg} \text { of } \mathrm{Po}_{4}\end{array}$ \\
\hline $1.6-2.1$ & $0.8-0.16$ & $0.16-0.24$ \\
\hline $1.1-1.5$ & $0.16-0.24$ & $0.24-0.30$ \\
\hline$<1.1$ & $0.24-0.30$ & 0.50 \\
\hline
\end{tabular}

Oral Repletion: Serum Phosphate > 1mg./dL, 1-2 g (32-64mmol) of Phosphorus/ day divided in 3 - 4 doses.

Suggestions for Magnesium Replacement ${ }^{31}$

\begin{tabular}{|c|c|}
\hline Condition & Magnesium - dose \\
\hline Daily requirement & 0.4m Eq/Kg./day \\
\hline Mild to Moderate hypomagnesemia & Initial 1m Eq/Kg over 24 Hrs. \\
\hline (Serum level $1.2-1.7 \mathrm{mg} / \mathrm{dl}$ ) & Follow : $0.5 \mathrm{mEq} / \mathrm{Kg}$ over $24 \mathrm{Hrs} . \mathrm{x} 5$ days \\
\hline \multirow[t]{3}{*}{ Severe Hypomagnesemia (Serum level < 1.2mg/dl) } & Initial : 2g over 2 minutes \\
\hline & Follow : 5 g over 6 hours. \\
\hline & Follow : 5 g over 12 hrs. $\mathrm{x} 5$ days \\
\hline
\end{tabular}

Suggestions for Potassium Replacement (If serum potassium $<3.0 \mathrm{mmol} / \mathrm{L}$ )

Administer: Potassium 0.3mmol/kg/hr for 4-6 hrs. Then 2-4mmol/kg/day; monitor ECG for arrhythmias during replacement therapy

If very low plasma electrolyte values are demonstrated e.g. phosphate $<0.32 \mathrm{mmol} /$, Potassium is $<2.5 \mathrm{mmol} / \mathrm{l}$ and Magnesium is $<0.5 \mathrm{mmol} / 1$, then the institution of feeding or nutritional support may result in a further drop of these electrolytes to possibly critical levels. Electrolyte correction with oral/I.V. supplementation is required to achieve levels above these thresholds before institution of feeding.

\section{Correction of Hypoglycemia:}

Monitor blood glucose once to twice daily unless more frequent tests are indicated (i.e. for those patients with known diabetes or IGT).

If hypoglycemic replace intravenous fluids with $5 \%$ glucose.

\section{Management of Hypothermia:}

Monitor body temperature, and if necessary record temperature at least daily.

Hypothermia is commonly associated with malnutrition. It's correction should be simultaneously with fluid rehydration and can include provision of hot drinks and blanket.

Correction of micronutrient deficiencies

Administer thiamine 1-2mg/kg body weight IM. $100 \mathrm{mg}$ IV should be administered in 50-100 ml 5\% dextrose over 30 min., prior to refeeding as stat dose. Repeat the thiamine daily for 48 hours then weekly.

Administer vitamin B complex IV until oral route is available.

\section{Commencing feeding in patients at risk of refeeding syndrome}

Provision of calories should be done gradually during first 5- 7 days; 20Kcal-25Kcal/Kg/Day (57 days) then after 7 day 25-35 Kcal./kg./day. ${ }^{4}$ or 
no more than $20 \%$ above BEE. ${ }^{11}$ or $1000 \mathrm{Kcal} /$ day. ${ }^{4}$ or $50 \%$ of total needs based on HBEx1.31.5. 32

For every 200-250 Kcal it is necessary to provide $6.25 \mathrm{~g}$ Protein, $1.8 \mathrm{mmol} / \mathrm{Ca}^{++}, 2.9 \mathrm{mmol}$ Phosphorus, 1.0mmol Magnesium, 10mmol Potassium, 7mmol Sodium and Chloride.

At the sight of a severely malnourished patient, the clinician should avoid the temptation to initiate excessive amount of nutrition support, rather, begin nutrition repletion slowly. While it is appropriate to start nutrition intervention as soon as feasible, it is dangerous to provide excessive calories or fluids to patients whose body has adapted to starvation.

In initial period $20 \mathrm{Kcal} / \mathrm{Kg}$. should be given to severely malnourished patients. A mixed fuel source of protein, fat and carbohydrate should be provided. Glucose infusion should not exceed 150200 grams per day or $2 \mathrm{mg} / \mathrm{kg} / \mathrm{min}$ initially. ${ }^{11}$ Protein can generally be initiated at full goal. (Up to 1.5g/ Kg/day). ${ }^{11}$ For estimating nutritional needs, the patients actual weight (or adjusted if obese) should be used rather than an ideal weight, which may be over (or under) estimate needs.

\section{Conclusion}

In many cases, nutrition support is a life saving modality. However, if nutrition is not delivered properly, Re feeding Syndrome represents a significant risk of morbidity and mortality in malnourished patients. Meticulous attention to the replacement of electrolyte imbalances and slow re-introduction of calories and protein will decrease the risk of cardiac and respiratory failure. Prospective, randomized controlled trials are needed to better identify patients prone to complications from Refeeding Syndrome. This, in turn will help to determine the best approach to Refeeding the malnourished patients.

\section{Authors Affiliation}

1. * Dr. Raghuveer Choudhary, Assistant Professor, Dept. of Physiology, Dr S.N. Medical College, Jodhpur (342001) Rajasthan, India. 4F/54,New Power House Road ,Jodhpur 342001 (Raj.) India. Email: drraghu74@yahoo.com, Cell: +91-9829216643

2. Dr. Vinode Kumar Chawala, Professor, Dept. of Physiology, Dr S.N. Medical College, Jodhpur (342001)Rajasthan, India.

3. Dr. Jayant Kumar, Associate Professor, Dept. of Physiology, Dr S.N. Medical College, Jodhpur (342001) Rajasthan, India.

4. Dr Raj Kumar Rathore, Assistant Professor Dept. of Pharmacology, Dr S.N. Medical College, Jodhpur (342001)Rajasthan, India. * for correspondence

\section{References}

1 Ritz E. Acute hypophosphatemia Kidney Int. 1982; 22:84.

2. Schnitker MA, Mattman PE, Bliss TL: A clinical study of malnutrition in Japanese prisoners of war. Ann Intern Med. 1951; 35: 69.

3. Weinsier RL, Krumdieck CL: Death resulting from overzealous total parenteral nutrition: The Refeeding Syndrome revisited. Am J Clin Nutr 1981; 34: 393.

4. Solomon SM, Kirby DF. The Refeeding Syndrome: A review. JPEN 1990; 14: 90-7.

5. Sack GS, Walker J, Dickerson RN et al. Observations of hypophosphatemia and its management in nutrition support. Nutr Clin Pract 1994; 9 : 105-8

6. Gonzalez AG, Fajardo-Rodriguez A, Gonzalez Figueroa E. The incidence of the Refeeding Syndrome in cancer patients who receive artificial nutritional treatment (English abstract) Nutr Hosp 1996; 11: 98.

7. Hernandez - Aranda JC, Gallo-Chico B, Luna-Cruz ML et al. Malnutrition and total parentereal nutrition: A cohort study to determine incidence of the refeeding syndorme (English abstract). Rev Gastroentrol Mex 1997; 62: 260.

8. Crook MA, Hally V, Panteli JV. The importance of the Re feeding Syndrome. Nutrition 2001; 17 : 632-7.

9. Esphagan . Complications : Guidelines on Paediatric Parenteral Nutrition. J Paed Gastro Nutr 2005; 41:S1-S9.

10. Brooks MJ, Melnik G. The Refeeding Syndrome: an approach to understanding its complications and preventing its occurrence. Pharamacother 1995; 15(6): 713-26. 
11. Apovian CM, McMohan MM. Guidelines for refeeding the marasmic patient. Crit Care Med 1990; 18:1030-33.

12. Romanski S, Mc Mahon M. Metabolic acidosis and thiamine deficiency. Mayo clinic proceedings 1999; 74: 3 .

13. Wolfe RR. Regulation of glucose metabolism. In Burke JF ed. Surgical Physiology. Philadelphia: WB Saunders; 1983. p75-97.

14. BivinsBA, Hyde GL, Sachatello CR, et al. Physiopathology and management of hyperosmolar hyperglycemic nonketotic dehydration. Surg Gynecol Obstet 1982; 154: 534.

15. McMohan M, Manji N, Driscoll DF, et al. Parenteral nutrition in patients with diabetes mellitus: Theoretical and practical consideration. JPEN 1989; 13:545.

16. Hayek ME, Eistenberg PG: Severe Hypophosphatemia following institution of enteral feeding. Arch Surg 1989;124:1325

17. Thompson JS, Hodges RE: Preventing hypophosphatemia during total parenteral nutrition. JPEN 1984; 8: 137.

18. Knochel JP. The Pathophysiology and clinical characteristics of severe hypophosphatemia. Arch Intern Med 1977; 137: 203.

19 Havala T, Shronts E. Managing the complications associated with Refeeding Syndrome. Nutr Clin Pract 1990;5:23-9.

20. Matz R. Parallels between treated uncontrolled diabetes and the Refeeding Syndrome with emphasis on fluid and electrolyte abnormalities. Diabetes care 1994; 17: 1209-12.

21. Metheny NM. Fluid and Electrolyte Balance; nursing considerations. $4^{\text {th }}$ ed. Philadelphia:Lippincott PA; 2000. pp305-24.

22. Stacey M, SherrieW, Carol R. Much Ado About Refeeding. Practical Gastroenterlogy. Jan. 2005, 26-44.
23. Rindi G, Thiamin. In: Ziegler EE, Filer Jr. LJ, eds. Present knowledge in Nutrition. $7^{\text {th }}$ ed. Washington, DC: ILSI Press; 1996. p.160-164.

24. Doss A, Mahad D, Romanowski C. Wernicke encephalopathy: Unusal findings in nonalcoholics patients. J Computer Assisted Tomograpy 2003; $27: 2$.

25. Baughman Jr. F. Popp J. Wernicke's encephalopathy with intravenous hyperalimentation : remarks on similarities between Wernicke's encephalopathy and the phosphate depletion syndrome. The Mount Sinai J Med 1976; 43: 1.

26. Sauter P. Forgotten side effects of diuretics: lipids, glucose and vitamin B1 (thiamin) metabolism (English abstract). Scheweis Rundsch Med Prax 2004; 93: 20.

27. Ling ford - Hughes AR, Welch S, Nutt DJ, Evidenced based guide-lines for the pharmacological management of substance misuse, addiction and comorbidity: recommendations from the British Association for Psychopharmacology. J Psychopharmacol 2004; 18: 3.

28. Rubin MF, Narins RG. Hypophosphatemia: Pathophysiological and practical aspects of it's therapy. Semin Nephrol 1990; 10: 536-45.

29. Dicherson RN. Guidelines for the intravenous management of hypophosphatemia, hypomagnesemia, hypokalemia and hypocalcemia. Hosp Pharm 2001; 35; 1201-06.

30. Peppers M, Geheb M, Desai T. Hypophosphatemia and hyperphosphatemia. Crit Care Clin 1991; 7: 201-22.

31. Marino PL, The Hidden threat of magnesium deficiency. Crit care Med., 1991; 12 (6) 34-42, 4446.

32. Al Ateequi N, Allard J. Anorexia Nervosa: from starvation to refeeding. Clin Nutr Rounds 2001; 1(1): Available from http://www.cscn scne.ca/. 